\title{
Experiences of Filipino Pediatric Occupational Therapists in Batangas City on Telehealth
}

\author{
Rod Charlie Delos Reyes, Trisha Nicole Linang, Hazel Anne Magsombol, Mary Erleene Nebres, \\ Marianela Zoleta
}

\begin{abstract}
Background: Due to the onset of the COVID-19 pandemic, telehealth has become the safest, simplest, and most convenient way to deliver occupational therapy services amidst the outbreak. Objectives: The study aims to describe the experiences of pediatric Filipino occupational therapists in Batangas regarding telehealth. Methods: A descriptive qualitative design is utilized to achieve the objective of the study. The four (4) occupational therapists working in Batangas were interviewed via ZOOM Communications within 45 to 60 minutes using semi-structured question guidelines. All interviews were recorded and transcribed for thematic analysis. Results: Three themes emerged: perceived benefits of telehealth, enablers of telehealth, and challenges on telehealth. This summarized the experiences of the participants in the utilization of telehealth for occupational therapy service provision. Conclusion: Telehealth can be a permanent alternative service delivery model for occupational therapy, which creates opportunities for professional development also.

Index Terms - COVID-19, Occupational Therapists,

Qualitative Research, Telehealth.
\end{abstract}

\section{INTRODUCTION}

The World Health Organization (WHO) declared the 2019 coronavirus disease (COVID-19) a pandemic on March 11, 2020. The COVID-19 is linked to Severe Acute Respiratory Syndrome Coronavirus 2 (SARS-CoV) that similarly can be fatal. (1) This disease is transmitted through direct, indirect, or close contact with infected people via infected secretions such as saliva and respiratory secretions or their respiratory droplets, which are expelled when an infected person coughs, sneezes, talks, or sings. (2) It received global attention from growing numbers of infected individuals and difficulties in eradicating it within communities. (3)

In the Philippines, there are 461,505 cases recorded as of December 21, 2020, with Batangas province having 11,352 confirmed cases that are still increasing. (4) Indeed, the global pandemic has disrupted everyone's daily life. The World Federation of Occupational Therapists (WFOT) recognizes

Rod Charlie Delos Reyes, MSOT, OTRP, College of Allied Medical Sciences, University of Batangas and The Graduate School, University of Santo Tomas Manila, Philippines

Trisha Nicole Linang, College of Allied Medical Sciences, University of Batangas, Batangas City, Philippines

Hazel Anne Magsombol, College of Allied Medical Sciences, University of Batangas, Batangas City, Philippines

Mary Erleene Nebres, College of Allied Medical Sciences, University of Batangas, Batangas City

Marianela Zoleta, College of Allied Medical Sciences, University of Batangas, Batangas City, Philippines the consequences of the COVID-19 pandemic on how people engage in their daily activities and occupations as a result of disruptive changes in community access, resource availability, and individual health and well-being. (5) Hence, with the limited activities and restricted physical interactions due to the imposed varied levels of community quarantines, the COVID-19 has brought significant effects to the global economy and challenged the health care systems. In response to these situations, occupational therapists are forced to adopt the new normal in delivering therapy services, including telehealth. (6) The same scenario applies to the occupational therapists in the Batangas province.

Telehealth is defined as the remote delivery of a variety of health care and rehabilitative services for persons with physical and cognitive disabilities, and medical conditions through telecommunication technology. (7) Furthermore, as stated by the Health Resources and Services Administration, it is the use of information, communications, and technology to promote the distant delivery of services among patients and healthcare practitioners. (8) being called the social media capital of the world, the Philippines continues to face challenges to the adoption of telerehabilitation arising from the stakeholder or the environment. (13)

With the outbreak of COVID-19, the country shifted and adopted Telehealth, an alternative model of delivering health services to clients. This model was utilized to prevent the spread and risk of acquiring the virus. Despite being perceived as the most practical and safest alternative mode of continuing service delivery, the identified technological problems in the country must be considered for these may interfere with the service provision.

In line with the shift, the Philippine Academy of Occupational Therapists, Inc. (PAOT) promulgated the Interim Guidelines on the Practice of Occupational Therapy amidst the COVID-19 situation in the Philippines. (14) This document was released on the 16th day of March 2020. The guideline was focused on the use of telehealth as a form of occupational therapy service provision. Telehealth, being the most appropriate service delivery model during this global crisis, was adopted by the profession. It was defined by the organization as an alternative form of service provision given when the therapist and the client are in different physical locations. (14) In this platform, service provision can be synchronous, asynchronous, or a combination of both. (15) However, despite variance in service delivery, the same standard of care should be met. Hence, continuous monitoring is then required to determine whether this platform is effective and efficient as an alternative service 
delivery model. That being said, ways to evaluate and assess telehealth is essential.

The unforeseen pandemic then drastically accelerated the uptake and adaptation of telehealth. Telehealth became an alternative to the traditional health care system as it helps meet patients' demands of greater flexibility, giving them ways to connect regardless of their location and whatever need arises, especially in this trying time. (16) However, there are conveyed tones on the need to assess the service delivery model towards its efficacy for the stakeholders and one's knowledge, attitude, and skill on telehealth.

Consequently, the authors aimed to determine the experiences of occupational therapists working in Batangas with regards to telehealth to abide by the proposed continuous evaluation and monitoring of the practice and essentially look into its real impact on the local Philippine health care system in delivering occupational therapy services.

\section{MATERIALS AND METHODS}

\section{A. Study Design}

In order to describe the experiences of Filipino occupational therapists working in Batangas regarding their telehealth practice, a descriptive qualitative method was utilized. This method provided guidance to explore and understand their perspectives regarding the new model of service delivery.

\section{B. Data Collection}

Data for the study were taken through semi-structured in-depth interviews with each participant. Upon the confirmation to participate, the schedule and meeting details were sent through electronic mail. The interview was conducted through the Zoom Communications platform since it is an easy to use and secure online platform for meetings. (20) Each meeting lasted from 45 to 60 minutes with the last four authors present along with the participant. One of the present authors facilitated the discussion by asking the participants questions related to their experiences in telehealth practice, while the others take notes, observe the interview, and manage the platform. Some of the questions include: "What are your experiences with telehealth?", "What are the common concerns you encounter during telehealth?", and "What are your perceived benefits or advantages of telehealth?".

\section{Data Analysis}

The interviews were recorded. Each was transcribed by the second and third authors, followed by validation by the fourth and fifth authors. This qualitative study utilized these narratives from the interview to explore and understand the experiences of occupational therapists in Batangas regarding their telehealth practice. The qualitative data were analyzed through thematic analysis. The analysis was done by (1) familiarizing with the narratives through reading, (2) coding through noting repeated words and phrases, (3) generating and reviewing themes based on the codes gathered, and (4) defining the themes after quorum among the authors. (21) To address potential biases, reflective commentaries were utilized by bringing into awareness the assumptions and opinions of each author while analyzing the data. (22)

\section{RESULTS}

The study was able to explore the experiences of at least four (4) occupational therapists working in Batangas within private therapy centers while using telehealth as a model of service delivery. Their demographics are shown in Table 1

Table 1. Participants' Demographics

\begin{tabular}{|l|l|l|}
\hline Participant & Gender & Years of Experience \\
\hline P1 & Male & 3 \\
\hline P2 & Female & 6 \\
\hline P3 & Female & 3 \\
\hline P4 & Female & 10 \\
\hline
\end{tabular}

After the analysis of the narratives taken during the interview, three (3) themes emerged: perceived benefits, enablers of telehealth, and challenges of telehealth. Each theme was supported by different codes. See Table 2.

Table 2. Experiences of Pediatric Occupational Therapists in Batangas

\begin{tabular}{|l|l|l|}
\hline Theme 1 & Theme 2 & Theme 3 \\
Perceived Benefits & Enablers of Telehealth & Challenges of Telehealth \\
\hline 1.1 Continuity of Care & 2.1 Need to Adapt & 3.1 Technological Concerns \\
\hline
\end{tabular}




\begin{tabular}{|l|l|l|}
\hline 1.2 Accessibility of Care & 2.2 Positive Attitude & 3.2 Availability of Resources \\
1.3 Increased Parent Involvement & & 3.3 Workload Demand \\
1.4 Professional Development & & 3.4 Apprehension on Telehealth \\
& & 3.5 Client-specific \\
& & 3.6 \\
& & 3.7 Reliability Concerns \\
& & 3.8 Behavioral Problem \\
\hline
\end{tabular}

\section{DISCUSSION}

\section{A. Perceived Benefits}

This first theme presents the perspectives of occupational therapists about the benefits of telehealth in the delivery of therapy services. It is perceived to enable continuity and accessibility of care, increased parent involvement, and pushes professional development. The utilization of telehealth in the Philippines made it possible for clients to avail and receive treatment despite the on-going pandemic. One expressed how telehealth greatly assisted clients in this time of the pandemic:

“...therapy is a very essential service....so therapy should not be stopped just because there is a pandemic.... it's better to have services like this...than none." (P4)

COVID-19 triggered the regression of many children since various changes in their routines and limited activities and physical interactions may overwhelm them. (23) Moreover, in-person therapy services are not possible. Therapy services help children in improving their skills and behaviors. (24) The absence of these services may lead to regression which can interfere with the client's improvement and development. This is further strengthened by the claims of the participants:

"Greater harm may arise, if you [occupational therapist] will not be able to treat him...although it [telehealth] has limitations, at least you were able to see the client and address his concerns." (P4)

"...I am afraid that my kids will regress and also to give help despite all the happenings in this pandemic setting." (P3)

Moreover, it was emphasized that safety protocols such as proper use of PPEs, physical distancing, and proper hygiene prevent the spread of the virus. (25) With all the safety protocols mandated by the government in preventing the spread of COVID-19, telehealth made it possible for clients to acquire therapy sessions. Clients have the chance to set and schedule appointments easily at the comfort of their homes without bothering to travel. One participant remarked:

"Teletherapy makes it possible for clients who have physical disabilities, geographic location issues or scheduling issues to receive interventions." (P2)

Adoption of telehealth ensures that clients will be able to acquire occupational therapy services despite the pandemic, social distancing, and all the protocols. Some said:

"We have clients who have to travel 2 to 3 hours to reach the center. But through telehealth, they do not have to travel here just to receive therapy..." (P4)

"This platform has no geographical boundary so you can access clients from any place even from outside of the country... it helps clients to save money on treatment...clients have fewer expenses because they do not have to travel." (P2)

Another perceived benefit is that compared to in-person sessions, parents have more involvement in telehealth because they supervise and guide their children during therapy sessions.

"Since the parents are beside their kids during the telehealth session, they were able to see how it is done and the actual performance of their kids,.... since they are in-charge in preparing the materials for the session and they need to be beside their kids during therapy..." (P4)

Aside from its benefits to the clients, telehealth helped the occupational therapists to challenge their ability to adapt in these challenging times. Occupational therapists learned many new skills as they try to adapt and provide therapy services through telehealth. Communication between colleagues heightens professional development because they help each other in familiarizing, coping, and adjusting with this alternative service delivery model. Some of the opinions of the participants are:

"Familiarizing myself with various software programs and applications that are convenient to use....I enjoyed making creative presentation slides that not only entertain my students but also facilitate their learnings as well." (P2)

"Usually, we use Skype to do counseling. It [Zoom Communications] was something new to me and when I started [using it] I asked my colleagues how do you do this, or can we practice? That's how I started with using Zoom, and then eventually I got used to it." (P1)

\section{B. Enablers of Telehealth}

In the second theme, participants emphasized the factors that facilitate the delivery of telehealth service. These are the 
factors that they had experienced for the past months. The two important matters that support their practice are the need to adapt and the positive attitude of stakeholders.

Similar to the other developing countries, there is an urgent need in adopting telehealth in the Philippines due to COVID-19. (13) The global health crisis was the main reason that made the participants pursue telehealth. The majority of the participants were doing telehealth for the first time in occupational therapy practice. This encapsulates that the participants ensure practicality and following health safety protocols during a pandemic while delivering occupational therapy service. One participant claimed:

"We do not know when this pandemic will end. Other centers, we thought after 2 or 3 months, it would be okay but look, it's already been 7 months, it's the same situation. That's why everyone was pushed to try telehealth." (P4)

Along with this need to adapt, the positive attitude of parents towards the provision of telehealth service enables telehealth service delivery. As stated by the participants, the parents or caregivers must be open to this alternative model of delivery service to facilitate the provision of treatment and healthcare services. To successfully administer telehealth, parents should be willing to attend and assist their children during the session as well as in preparing the materials needed for the session.

\section{Challenges of Telehealth}

This last theme presents the obstacles and troubles experienced by occupational therapists in delivering occupational therapy services through telehealth. Within this theme are eight factors that post a threat to the practice: technological concerns, availability of resources, workload demand, apprehension on telehealth, client specificity, amount of supervision, reliability concerns, and behavioral problems.

Internet disconnection was the most reported problem and prevalent theme that occurs in both the therapist and the client. The American Academy of Allergy Asthma \& Immunology states that the basic requirement for telehealth is a secure broadband internet connection. It requires a stable internet connection to access optimal service that may have otherwise been limited due to service provider shortages and inclement weather. (26) Along with internet disconnections, the availability of resources also poses challenges in telehealth practice. All the participants reported that they don't have problems with the resources they use during the provision of telehealth like the devices, internet connections, toys, and the likes however, they expressed that the lack of resources in their clients limits the delivery of occupational therapy service. Technology has been the focus to support telehealth and includes devices such as laptops and smartphones. Participants need to consider the availability and accessibility of clients with technology before the provision of telehealth service. One participant said:

"The availability of devices in their client's end also affects the frequency of the delivery of telehealth service. It is also a problem when the sibling of the client is studying. For example, there is a conflict in the schedule of the therapy, since they only have one laptop, they need to share it". (P4)

Furthermore, with the majority of participants using telehealth for the first time, there have been adjustments with the transition of in-person sessions to virtual therapy. The increased demands in preparation of activities theme echoed the participants' preparation before telehealth sessions. This encompasses the participants' adjustments and familiarization with software programs and applications.

"Then teletherapy is also demanding in the preparation of activities that must be said to the parent one to two days before the session so that parent can ready the needed materials, then preparation of activities can be time-consuming that's the drawback also... I tend to rearrange and modify the sequence of activities according to their preferences." (P2)

Also, participants indicated that they are afraid and hesitant in pursuing telehealth due to lack of structure and background to it. One participant blurted out:

“At first, I'm not willing to try it. I'm afraid. I thought of many things, for example, my kid might also have a hard time." (P4)

In terms of the stakeholder, the participants of the study pointed out that the major disadvantage of telehealth is that it is not for everyone. Teletherapy can provide alternative doors for treatment but it is not the same as a brick and mortar of a real therapy session. Aside from the limited resources and space, not all can access teletherapy, nor not all children can benefit from telehealth. Some clients may have the capacity to continue availing and receiving therapy sessions but, there are also some clients who are having a hard time accessing telehealth due to some technology-related issues and financial problems. A participant summarized this theme with:

"Telehealth is not everyone's cup of tea." (P1)

The amount of supervision from the parent is very important especially in synchronous interaction with the occupational therapist. Since telehealth is mostly administered through digital means, there is a great need for facilitators who will be collaborating with the therapist in providing therapy at home. Despite its benefit of involving the parent or caregiver, it is too demanding also on the part of the parent or caregiver. Supervision is important especially to children who have difficulty adapting and complying with the new mode of delivery service and an adequate amount of supervision is ideal for the telehealth service to be effective. It was observed by one participant:

"There are kids that need to be supervised by someone, but then sometimes parents are busy on their job or household chores and they can't supervise the kid." (P3)

Although there are stakeholders that are positive about telehealth, there are still some people that have resistance to trying something new.

"There are some parents who are not aware of the positive outcome that the telehealth would provide for their kids and since some have financial difficulties there are indecisive to 
give teletherapy a try." (P2)

And lastly, experiences of the participants in dealing with their clients' behavioral problems during the administration of telehealth services can be understood by the following statements:

"Since it is a new set-up, many kids have difficulty adapting to sudden changes. During our in-person sessions, their performance is very good but when it comes to this new set-up, they tend to be confused about why they need to see their teacher on screen. So, the kids also have behavioral difficulties, sometimes they cannot attend well." (P4)

Behavioral problems such as inattention, distractibility, difficulty to adapt, task avoidance, and tantrums are challenges that usually manifest during the first week of provision but resolve upon the establishment of telehealth.

\section{CONCLUSION}

With the sudden outbreak of COVID-19, Filipino occupational therapists felt the need to transition from in-person sessions to the utilization of telehealth. As telehealth is a novel mode of delivering occupational therapy service, Filipino occupational therapists experience challenges in the use of telehealth. Nevertheless, telehealth is perceived to be beneficial for both clients and occupational therapists as it provides continuity of care to clients, accessibility to occupational therapy service, and parent's involvement toward therapeutic processes increases. Professional development of occupational therapists also emerged since they learn and develop new skills in the administration of telehealth.

\section{REFERENCES}

[1] Meng L, Hua F, \& Bian Z. Coronavirus disease 2019 (COVID-19): emerging and future challenges for dental and oral medicine. J. of Dental Research. 2020

[2] World Health Organization [Internet]. Transmission of SARS-CoV-2:implications for infection prevention precautions. WHO. [Updated 2020] Available from: https://www.who.int/news-room/commentaries/detail/transmission-ofsars-cov-2-implications-for-infection-prevention-precautions

[3] Guo Y, et al. The origin, transmission and clinical therapies on coronavirus disease 2019 (COVID-19) outbreak - an update on the status. Mil Med Res. 2020. Mar;7(1):11

[4] Department of Health [Internet]. Covid-19 tracker. Govph. [Updated 2020] Available from https://doh.gov.ph/covid19tracker

[5] World Federation of Occupational Therapists [Internet] Public statement - occupational therapy response to the COVID-19 pandemic. WFOT. [Updated 2020] Available from https://wfot.org/about/public-statement-occupational-therapy-response -to-the-covid-19-pandemic

[6] Sy, M. et al. Shared voices of Filipino occupational therapists during the COVID-19 pandemic: Reflections from an online forum. World Federation of Occupational Therapists Bulletin, 2020 May18;76(1), 60-64.

[7] Manenti R, et al. Effectiveness of an Innovative Cognitive Treatment and Telerehabilitation on Subjects With Mild Cognitive Impairment: A Multicenter, Randomized, Active-Controlled Study [Internet]. Frontiers. Frontiers; 2020 [cited 2020Dec22]. Available from: https://www.frontiersin.org/articles/10.3389/fnagi.2020.585988/full

[8] Office for Civil Rights-US Department of Health and Human Services. 3015-What is telehealth? [Internet]. HHS.gov. 2020 [cited 2020Dec22]. Available from: https://www.hhs.gov/hipaa/for-professionals/faq/3015/what-is-teleheal th/index.html

[9] Investopedia. Top 25 Developed and Developing Countries [Internet] Investopedia. Investopedia; 2020 [cited 2020Dec22]. Available from: https://www.investopedia.com/updates/top-developing-countries/

[10] Fernandez-Marcelo P.G., Ho B.L., Faustorilla J.F., Evangelista A.L., Pedrena M., Marcelo A. Emerging eHealth directions in the Philippines. Yearb Med Inform. 2012;7:144-152.

[11] Leyco CS. Filipinos pay more for slow Internet services - World Bank [Internet]. Manila Bulletin. 2020 [cited 2020 Dec22]. Available from https://mb.com.ph/2020/10/06/filipinos-pay-more-for-slow-internet-se rvices-world-bank/

[12] Rodriguez M. Why Exactly is the Internet so slow in the Philippines? [Internet] SPOT.PH. [Updated 2020] Available from: https://www.spot.ph/newsfeatures/the-latest-news-features/83631/inte rnet-speed-philippines-why-is-it-slow-a4362-20200916-lfrm

[13] Leochico CFD, Espiritu AI, Ignacio SD, Mojica JAP. Challenges to the Emergence of Telerehabilitation in a Developing Country: A Systematic Review [Internet]. Frontiers in neurology. Frontiers Media S.A.; 2020 [cited 2020Dec22]. Available from: https://www.ncbi.nlm.nih.gov/pmc/articles/PMC7505991/

[14] Philippine Academy of Occupational Therapy [Internet]. PAOT Interim Guidelines on the Practice of Occupational Therapy.[Updated 2020] Available from: https://www.wfot.org/assets/resources/PAOT-Interim-Guidelines-on-t he-Practice-of-OT-amidst-the-COVID-19-situation-May-2020.pdf

[15] Cason J. Telehealth: A Rapidly Developing Service Delivery Model for Occupational Therapy [Internet]. International Journal of Telerehabilitation. [cited 2020Dec22]. Available from: https://telerehab.pitt.edu/ojs/index.php/Telerehab/article/view/6148/6 486

[16] Morgan M. Time's Running Out: Why Providers Must Adopt Telehealth in 2020 [Internet]. Healthcare IT News. 2019 [cited 2020Dec22]. Available from: https://hitconsultant.net/2019/12/27/times-running-out-why-providersmust-adopt-telehealth-in-2020/

[17] Kim H, Sefcik JS, Bradway C. Characteristics of Qualitative Descriptive Studies: A Systematic Review. Research in Nursing \& Health. 2017;40(1):23-42.

[18] McCombes S. Descriptive Research Design: Definition, Methods and Examples [Internet]. Scribbr. 2020 [cited 2020Dec22]. Available from: https://www.scribbr.com/methodology/descriptive-research/

[19] Lavrakas, P. Purposive Sample. [Internet].Sage Research Methods. [cited 2020Dec23]. Available from: https://methods.sagepub.com/reference/encyclopedia-of-survey-resear ch-methods/n419.xml. (2008).

[20] Archibald, M. Using Zoom Video conferencing for Qualitative Data Collection: Perceptions and Experiences of Researchers and Experiences of Researchers and Participants. [Internet]. SAGE Journals. [cited 2020Dec22]. Available from: https://journals.sagepub.com/doi/full/10.1177/1609406919874596

[21] Caulfield J. How to Do Thematic Analysis: A Step-by-Step Guide \& Examples [Internet]. Scribbr. [cited 2020Dec22]. Available from: https://www.scribbr.com/methodology/thematic-analysis/

[22] Chown, G, et. al. Phenomenological study on the perception of occupational therapists practicing in the emergency department. Open $\mathrm{J}$ Occup Ther. 2016

[23] Clopton J. Pandemic Causing Kids to Regress, Experts Say [Internet] WebMD. WebMD; 2020 [cited 2020Dec22]. Available from: https://www.webmd.com/lung/news/20200715/pandemic-causing-kids -to-regress-experts-say

[24] Cullinan CC, editor. Taking Your Child to a Therapist (for Parents) Nemours KidsHealth [Internet]. KidsHealth. The Nemours Foundation; 2018 [cited 2020Dec22]. Available from: https://kidshealth.org/en/parents/finding-therapist.html

[25] Department of Health [Internet]. DOH Issues Press Release: Safety Prevention Protocols still Best Defense Against COVID-19. [Updated 2020]. Available from: https://doh.gov.ph/node/23315

[26] O'Dowd E. [Internet]. Lack of broadband access can hinder rural telehealth programs. [Updated 2018]. Available from: https://hitinfrastructure.com/news/lack-of-broadband-access-can-hind er-rural-telehealth-programs 